\title{
Primary Cemented Hemiarthroplasty in Unstable Inter- Trochanteric Fracture of Femur in Elderly
}

\author{
Varun Goel $^{1 *}$, Neha Bhardwaj ${ }^{2}$ and Abhishek Garg ${ }^{3}$ \\ ${ }^{1}$ Department of Orthopedics, PGIMS, India
}

${ }^{2}$ Adesh institute of Medical Sciences and Research, India

${ }^{3}$ Department of orthopedics, PGIMS, India

*Corresponding author: Dr. Varun Goel, Doctors's Hostel, Department of Orthopaedics, PGIMS, Rohtak, Haryana, India

\begin{abstract}
Introduction: Unstable Intertrochanteric fractures are Complex fractures which become more difficult when it's associated with age related osteoporosis. Internal fixation methods like Dynamic hip screws and proximal femoral nails have lot of complications which adds to the morbidity and mortality of the patient.

Material and Methods: 23 patients aged more than 60 years presenting with unstable Intertrochanteric Femur fracture were operated with primary cemented bipolar Hemiarthroplasty Along with Greater Trochanter and Lesser Trochanter reconstruction with Steel wires or non-absorbable sutures.

Result: The average Harris Hip Score (HHS) at final follow up was 85.24 with 9 excellent, 7 good, 3 fair and 2 poor results.

Conclusion: Hemiarthroplasty in unstable Intertrochanteric fractures is better than other methods of internal fixation in elderly patients as it has less complications and the major advantage of bipolar Hemiarthroplasty is early mobilization and less mechanical complications.
\end{abstract}

Keywords: Bipolar; Hemiarthroplasty; unstable Intertrochanteric fracture; osteoporosis

\section{Introduction}

Around $45 \%$ of all hip fractures are Intertrochanteric fractures (IT fractures) [1] and the incidences of hip fractures are still higher in elderly. With increase in life expectancy, there is an increase in population of elderly patients. Osteoporosis which is very common in elderly, is a major cause of fractures following trivial trauma. About $35-40 \%$ of Intertrochanteric fractures are unstable [2]. The treatment of stable IT fractures is osteosynthesis with good results but there has been a wide consensus regarding the best treatment modality for unstable Intertrochanteric fractures in elderly patients. The major problem with these fractures is difficulty in obtaining acceptable reduction, poor bone quality and high rates of morbidity and mortality due to various factors especially prolonged recumbency. The primary goal of treatment is stable fixation and early mobilization. The treatment of these fractures evolved from conservative treatment with the skeletal traction to operative procedures like fixed angle blade plates, sliding hip screws and intramedullary devices. [3] Hemiarthroplasty and Total Joint Replacement is mainly used for femoral neck fractures and as a salvage for failed pinning and other complication of primary surgical procedure [4]. osteosynthesis in unstable Intertrochanteric fractures with osteoporosis has failure rate between $4-16.5 \%$. [5] When internal fixation is adopted, the range of general complications such as Deep Venous Thrombosis (DVT), Pulmonary embolism, Pneumonia, bed sores etc. ranges from 22-50\%. [6-7] The primary aim of this study is to analyze the role of primary cemented Hemiarthroplasty in unstable Intertrochanteric fracture of femur in elderly patients.

\section{Material and Methods}

The study was done prospectively on 23 patients aged more than 60 years presenting with unstable Intertrochanteric Femur fracture. One of the cases died on 8th month postoperatively and one case was lost to follow up after 4th month postoperatively. These 2 cases were excluded and only 21 cases were included in the study. The fractures were classified according to AO/OTA and Evan's classification and the fractures with A0/OTA type 31-A2.2 
and 31-A2.3 and Evan's type 3 and 4 were included in the study. Only the patients who were independently mobile before sustaining injury were included in the study were included. Written informed consent was taken from all the patients. The patients who were unfit for surgical procedure or polytrauma patients, open or pathological fractures, old or malunited fractures were excluded from the study. The patients who had any significant rheumatological, neurological or psychiatric disorders were also excluded from the study.

\section{Surgical Technique}

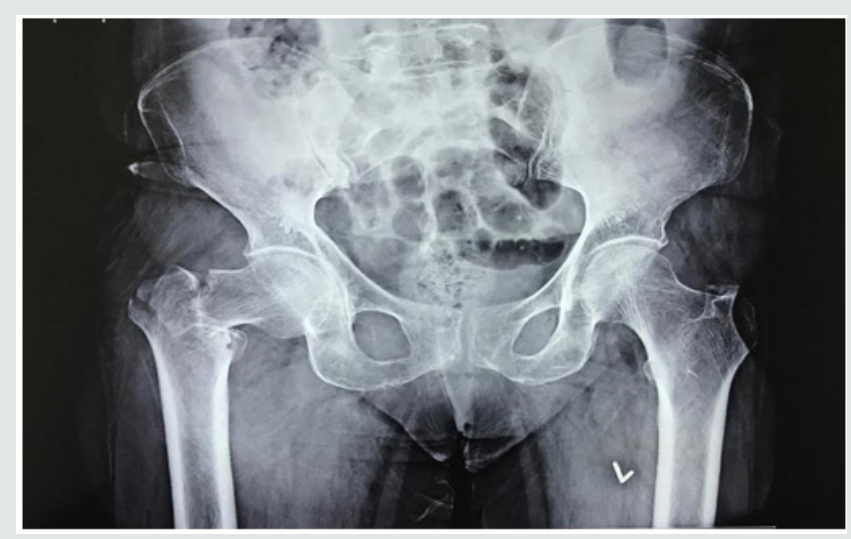

Figure 1: Preoperative $X$-ray.

Primary cemented bipolar Hemiarthroplasty was done using posterolateral approach in lateral position. The joint capsule opened using inverted ' $\mathrm{T}$ ' capsulotomy incision and then the fracture pattern assessed. The femoral head was then extracted by cutting the head of femur from neck, at sub capital region. Then the Greater Trochanter (GT) and Lesser Trochanter (LT) were temporarily reconstructed according to their comminution and displacement. Then excessive part of neck trimmed, and femoral canal prepared with reamers and broaches. Trial stem was then inserted taking care of appropriate version of prosthesis, by taking the long axis of leg and transcondylar axis of lower end of femur as reference. In severely comminuted fractures, especially in comminuted LT fractures, exact prosthesis height determination was difficult. So, both the knees were brought together, and height of prosthesis was marked. Second generation cementing technique and cement restrictor was used in all the cases. Appropriate prosthesis size selected, and the prosthesis stem was sunk till the previously marked point to avoid limb length discrepancy. Definitive reconstruction of GT and LT was then done using SS wires by Tension Band Wiring and/or encirclage technique. In cases of severe comminution Ethi bond sutures were used to keep Trochanter in place. There were a few cases where LT was severely comminuted, then it was left as it is within the soft tissue and the calcar was made using cement mantle. The sleeves of Gluteus Medius and vastus medialis anchored to GT using bone drills and wires. Short external rotators were also attached. The joint was then reduced; range of motion and stability was checked. Wound is then closed in layers over suction drain. Patients were allowed to sit on $1^{\text {st }}$ postoperative day onwards and standing with support was allowed on $2^{\text {nd }}$ postoperative day. Walking with support was allowed on $3^{\text {rd }}-4^{\text {th }}$ postoperative day and walking without support was encouraged and allowed as tolerated by patient. Follow up was done on $2^{\text {nd }}$ week, $4^{\text {th }}$ week, 3 months, 6 months, 1 year and 2 year. On each follow up clinic-radiological evaluation was done and Harris Hip Score (HHS) used for assessment. HHS less than 70 is graded as poor outcome, 70-79 as fair, 80-89 as good and 90-100 as excellent outcome. (Figures 1-3).

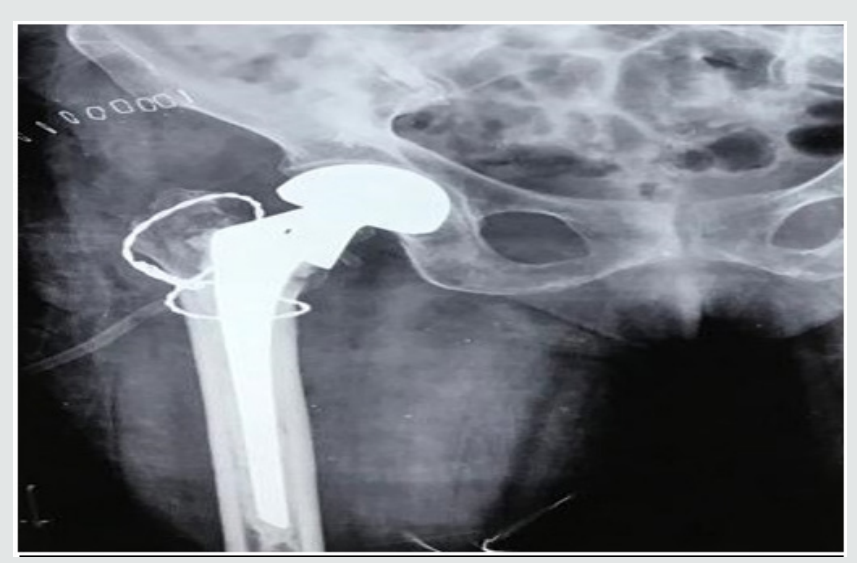

Figure 2: Immediate post-operative x-ray.

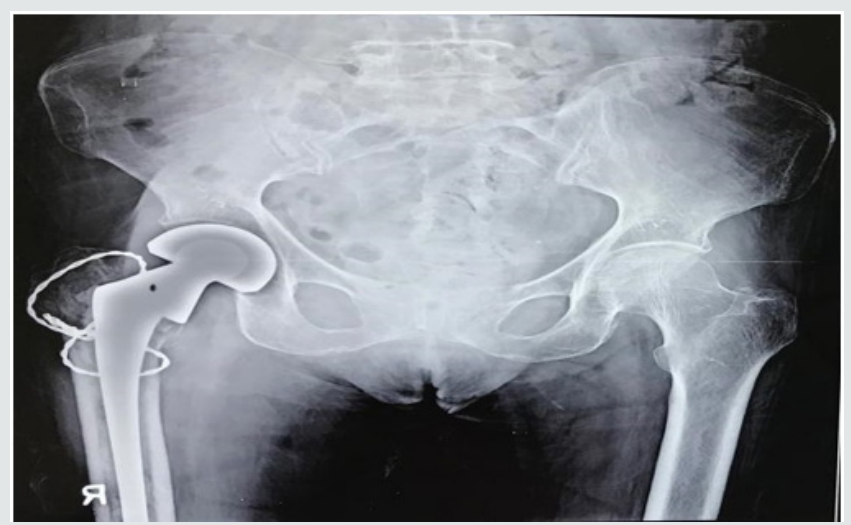

Figure 3: 1-year postoperative $x$-ray.

\section{Result}

Prospective study on 21 patients operated for unstable osteoporotic Intertrochanteric fractures with primary Hemiarthroplasty. The average age of the patients was 70 years and it included 14 male and 7 female patients. The duration of days since injury to presentation ranged between 1 to 13 days, average 4.33 days. The fracture pattern was of A0/OTA type A2.2 and A2.3 and Evan's type 3 and 4. The average duration of surgery was 89.76 minutes and average intraoperative blood loss was $426.19 \mathrm{ml}$. Postoperatively patient was made to bear weight on $1^{\text {st }}$ day to maximum on $8^{\text {th }}$ post-operative day (POD), average 3.52 days. Walking without support was started on $28^{\text {th }}$ to $62^{\text {nd }}$ day. There were 8 patients which were not able to walk without support. Five of them used stick to walk with limp, 2 used walkers and 1 patient refused to walk at all and was wheelchair bound and had poor result. Remaining 13 patients walked well without any support. The average hospital stay was 9.48 days with maximum of 25 days in a patient who developed bed sore on $13^{\text {th }}$ POD and was advised to stay in hospital for proper nursing care and dressing. There was 1 patient who developed superficial Surgical Site Infection (SSI) which 
was managed conservatively with antibiotics and dressing and one patient who developed deep SSI on $6^{\text {th }}$ week POD for which revision surgery had to be done with implant removal and re-implantation. There were 5 cases that had limb length discrepancies, 1 had limb lengthening and 4 had shortening which was managed with shoe raise but still continued to walk with limp and used walker/ stick. The average Harris Hip Score (HHS) at 3weeks was 74.29 and at final follow up average HHS was 85.24 with 9 excellent, 7 good, 3 fair and 2 poor results. All the patients were followed up for at least 1 year at maximum for 3 years 5 months; average follow up period was of 26.1 months.

\section{Discussion}

Management of Intertrochanteric fractures has always been a challenge. Every case is different, and no single surgical approach is applicable for all the patients. There are a number of surgeries proposed for Intertrochanteric fractures, either osteosynthesis by plates like Dynamic Hip Screws (DHS), intramedullary nails like Proximal Femoral Nail (PFN) etc. or replacement surgeries like Hemiarthroplasty or total hip replacement surgery. DHS and PFN are excellent implants which give very promising results, but all these also have high chances of morbidity due to mechanical failure and prolonged recumbence leading to conditions like pneumonia, bed sores, DVT etc. Since Intertrochanteric fractures are more common in elderly and these old patients are still more prone for such complication. Another challenge in elderly patients is Osteoporosis. Due to severe depletion of bone stock, the fixation is weak, poor screw hold, higher chances of cut outs and implant failure. Osteosynthesis with implants like DHS is easier and has better results with simple 2 parts Intertrochanteric fractures rather than comminuted unstable Intertrochanteric fractures. To avoid such complications in unstable Intertrochanteric fractures in elderly a good alternative is replacement of femoral head and neck with prosthesis, i.e. Hemiarthroplasty. In Intertrochanteric fractures doing Hemiarthroplasty is surgically more difficult as we have to repair Greater Trochanter (GT) and Lesser Trochanter (LT) also with steel wires or non-absorbable sutures but the surgery is very rewarding. In the above study we have done Hemiarthroplasty with Bipolar prosthesis with reconstruction of GT and LT with steel wires or non-absorbable sutures in 21 cases of unstable Intertrochanteric fractures in elderly patients. We had to make calcar with cement in a few cases where repair of LT was not possible.

The average age of the study patients was 70 years (ranging from 61-91 years). The patients presented after an average of 4.33 days after injury (ranging from 1-13 days). Postoperatively the patients were made to bear weight on average 3.52 days after surgery and walking with support allowed from $4^{\text {th }}, 5^{\text {th }}$ day onwards, with maximum delay of 28 days in one patient and another one patient refused to walk at all. Majority of the patients started walking after about 4 weeks, with exception of 8 cases which continued to use support for walking. Due to early mobilization of the patient there were very few complications and the hospital stay of the patients also drastically reduced. The average hospital stay was 9.48 days ranging from 7 days to maximum of 25 days in one patient. Study done by Thakur et al. [3] had an average age of study patients of 80.7 years and average duration of injury to surgery was 3 days. The patients were mobile with walker on average 2.6 days and average duration of hospital stay was 17.5 days. Another study done Ahmed Emory et al. [8] had average hospital stay period of study patients of 8.78 days and the mean time interval between injury and operation was 2.9 days. Out of 41 patients, 27 cases were able to ambulate independently using walker and 13 cases need assistance along with walker. Study done by Sachet et al. [9] has Harris Hip Score (HHS) at final follow up 84.8 +- 9.72 (58-97) with 10 excellent, 15 good, 7 fairs. 2 poor and 1 failed result. Choy et al. [10] in his study had mean HHS at last follow-up 80.6+- 9.3, with excellent in 8, good in 19 , fair in 9 and poor in 4 out of total 40 cases, i.e. better than fair results in $67.5 \%$ cases. In our study final mean HHS was 85.24 . out of total 21 cases, 9 had excellent results, 7 good, 3 fair and 2 poor results, i.e. $76.19 \%$ had better than fair results. In our study one patient had developed bed sore on $13^{\text {th }}$ post-operative day (POD), which healed with nursing care and dressing. This patient refused to walk and had poor results. There was one patient who developed superficial surgical site infection (SSI) which was managed conservatively with dressing and antibiotics and one patient developed deep SSI on $6^{\text {th }}$ week POD for which revision surgery was done with implant removal and reimplantation. This patient was a known diabetic with improper control of blood sugar. There were no cases of dislocation or loosening. Similar study done by Nikunkj et al. [1] on 28 patients of Intertrochanteric fractures had 1 patient with superficial SSI, 1 patient with deep SSI, 1 case of acetabular erosion, 4 patients of nonunion GT and 2 patients with GT cerclage wire breakage. There was 1 patient with periprosthetic fracture 6 months after surgery. Overall, he had mean HHS $84.8+-9.72$ at final follow up. Another study done by KV Puttakemparaju et al. [11] on 20 cases had 1 case of deep SSI for which implant removal was done, 1 case of grade 1 bed sore which healed with dressings and antibiotics. There was no dislocation, rotational deformities or subsidence of stem. Gashi et al. [12] in his study compared outcome of primary cemented bipolar Hemiarthroplasty with DHS in elderly patients with unstable Intertrochanteric fracture and concluded that early mobilization was significantly better in Hemiarthroplasty than DHS. General and mechanical complications were more common in DHS group. At final follow up mortality rate didn't differ in between the two groups but mean HHS was better in Hemiarthroplasty group. Comparative study done by Mohamad Emmi et al. [13] concluded that HHS (86+-9 vs. 75+-7.6), range of flexion (105+- 11 o vs. $90+-17$ ) , external rotation (35 +-7o vs. $20+-70$ ) were significantly higher in bipolar group compared to DHS group $(\mathrm{P}<0.05)$. However, there were no significant differences in pain severity in both the groups. Yee Suk Kim et al. [14] in a comparative study found that elderly patients with AO type A2 Intertrochanteric fractures, patients treated with Hemiarthroplasty were able to perform early ambulation. However, no significant difference in operative time, amount of postoperative transfusion, clinical results, hospital stay, and radiological failure rate was observed between bipolar Hemiarthroplasty and compression hip screw fixation groups. Study done by Sameer Ajit Mansukhani et al. [15] on DHS, Cemented bipolar Hemiarthroplasty and PFN for treatment of unstable Intertrochanteric fractures found that mean intraoperative blood was significantly less with PFN as compared with other two, whereas there was no significant difference in mean 
hospital stay and intraoperative time of all the three groups. There were more early complications seen in DHS group as compared to PFN and Hemiarthroplasty. Late complications such as femoral head AVN and implant cut outs were higher in PFN group whereas chances of hip dislocation were higher in Hemiarthroplasty patients. Mortality at 12 months was highest after bipolar Hemiarthroplasty and least after DHS. Reoperation rates were highest for PFN as compared to other two groups. On functional assessment unaided walking was best with DHS whereas other parameters didn't have any significant difference. He concluded that Treatment of unstable Intertrochanteric fracture of femur is a matter open to debate. Intertrochanteric fractures of elderly must be treated with considering the age of the patient, mental status, bone quality, and the type of fracture. It is certain that the main objective is to prevent the possible complications by early mobilization and to help the patient to return to their daily life. Kayali et al. [16] in their study found that cone Hemiarthroplasty can be an alternative treatment for unstable Intertrochanteric fractures in elderly patients so as to achieve earlier mobilization. The limitations of our study were that the sample size was small and there was no comparison of bipolar Hemiarthroplasty with osteosynthesis surgeries like DHS and PFN. The study period was not too long and so long-term complications like hip osteoarthritis, loosening, protrusion, stem failure etc. cannot be assessed.

\section{Conclusion}

Bipolar Hemiarthroplasty is a reasonably good alternative in unstable Intertrochanteric fractures in elderly patients who have severe osteoporosis. The major advantage of bipolar Hemiarthroplasty is early weight bearing and less short and midterm complications. Internal fixation is a preferred especially in young patients with stable Intertrochanteric fractures but have high complication rates in elderly with unstable fractures due to prolonged Recumbency and severe osteoporosis.

\section{References}

1. Maru N, Sayani K (2013) Unstable Intertrochanteric fractures in high risk elderly patients treated with primary bipolar Hemiarthroplasty: retrospective case series. Gujarat Medical Journal 68(2): 68-72.

2. Ukaj S, Gjyshinca B, Podvorica V, Ukaj F, Molliqaj G et al. (2017) Primary hemiarthroplasty for treatment of unstable pertrochanteric femoral fractures (AO/OTA Type 31 A2.3) in elderly osteoporotic patients. SICOT J, 3: 31 .
3. Thakur A, Lal M (2016) Cemented Hemiarthroplasty in elderly osteoporotic unstable Trochanteric fractures using fracture window. Malaysian Orthopaedic Journal 10(1): 5-10.

4. Stern MB, Goldstein TB (1977) The use of the Leinbach prosthesis in Intertrochanteric fractures of the hip. Clin Orthop Relat Res 128: 325331.

5. Haidukewych GJ, Berry DJ (2003) Hip Arthroplasty for salvage of failed treatment of Intertrochanteric hip fractures. J Bone Joint Surg Am 85(5): 899-904.

6. Kenzora JE, McCarthy RE, Lowell JD, Sledge CB (1984) Hip fracture mortality. Relation to age, treatment, preoperative illness, time of surgery, and complications. Clin Orthop Relat Res (186): 45-56.

7. Baumgaertner MR, Curtin SL, Lindskog DM (1998) Intramedullary versus extramedullary fixation for the treatment of Intertrochanteric hip fractures. Clin Orthop Relat Res (348): 87-94.

8. Elmorsy A, Saied M, Allah AA, Zaied M, Hafez M (2012) Primary Bipolar Arthroplasty in Unstable Intertrochanteric Fractures in Elderly. Open Journal of Orthopedics 2: 13-17.

9. Sancheti KH, Sancheti PK, Shyam AK, Patil S, Dhariwal Q et al. (2010) Primary Hemiarthroplasty for unstable osteoporotic Intertrochanteric fractures in the elderly: A retrospective case series. Indian J Orthop 44(4): 428-434.

10. Choy WS, Ahn JH, Ko JH, Kam BS, Lee DH (2010) Cementless Bipolar Hemiarthroplasty for Unstable Intertrochanteric Fractures in Elderly Patients. Clinics in Orthopedic Surgery 2(4): 221-226.

11. Puttakemparaju K V, Bes haj N R (2014) Unstable Intertrochanteric fracture in elderly treated with bipolar Hemiarthroplasty: A prospective case series.Afr J Trauma 3(2): 81-86.

12. Gashi YN, Elhadi AS, Elbushra IM (2018) Outcome of primary cemented bipolar Hemiarthroplasty compared with Dynamic Hip Screw in elderly patients with unstable Intertrochanteric fracture. Malaysian Orthopaedic Journal 12(1): 36-41.

13. Emami M, Manafi A, Hashemi B, Nemati A, Safari S (2013) Comparison of Intertrochanteric Fracture Fixation with Dynamic Hip Screw and Bipolar Hemiarthroplasty Techniques. Arch Bone Joint Surg 1(1): 14-17.

14. Kim YS, Hur JS, Hwang KT, Choi II-Y, Kim KH (2014) The comparison of compression hip screw and bipolar Hemiarthroplasty for the treatment of A0 Type A2 Intertrochanteric fractures. Hip Pelvis 26(2): 99-106.

15. Mansukhani SA, Tuteja SV, Kasodekar VB, Mukhi SR (2017) A comparative study of the Dynamic Hip Screw, the cemented Bipolar Hemiarthroplasty and the Proximal Femoral Nail for the treatment of unstable Intertrochanteric fractures. Journal of Clinical and Diagnostic Research 11(4): RC14-RC19.

16. Kayali C, Agus H, Ozluk S, Sanli C (2006) Treatment for unstable Intertrochanteric fracture in elderly patients: internal fixation versus cone Hemiarthroplasty. J Orthop Surg (Hong Kong) 14(3): 240-244.

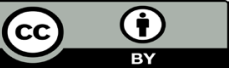

This work is licensed under Creative Commons Attribution 4.0 License

To Submit Your Article Click Here: Submit Article

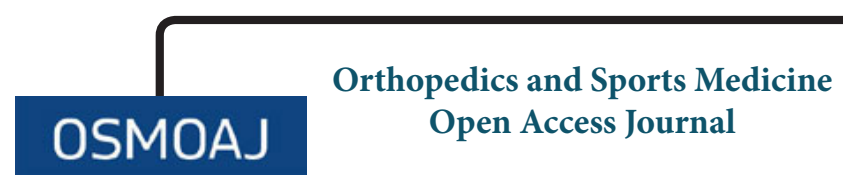

Assets of Publishing with us

- Global archiving of articles

- Immediate, unrestricted online access

- Rigorous Peer Review Process

- Authors Retain Copyrights

- Unique DOI for all articles 